\title{
Que peut-on faire en hydrologie avec un modèle conceptuel à un seul paramètre ?
}

\author{
How to use single-parameter conceptual model \\ in hydrology?
}

\author{
Claude Michel \\ Cemagref-Antony \\ Division Hydrologie-Hydraulique
}

\section{Introduction}

Le bassin représentatif de l'Orgeval est un exemple typique de petit bassin rural de la moitié nord de la France. D'une superficie de $104 \mathrm{~km}^{2}$, son observation a débuté en 1962 et a permis de nombreuses études et recherches, entre autres sur des modèles complexes. Dans ce qui suit, on recherche un modèle conceptuel global, aussi simple que possible, pour reconstituer les débits journaliers du bassin à partir des pluies et des températures.

\section{Démarche suivie}

Comme habituellement en matière de modélisation de bassins représentatifs et expérimentaux, on est parti initialement d'une modélisation relativement complexe. Pour l'Orgeval, il s'est agi tout particulièrement du modèle CREC à 9 paramètres (Galea, 1972, et Rifaat, 1980) du modèle CEQUEAU, indirectement du modèle non conceptuel DEJ $\emptyset$ REG (Tiercelin et Leviandier, 1977) et des essais d'amélioration de l'estimation de l'évaporation réelle (Oberlin, 1978). Outre la difficulté de mise en œuvre, la longueur des réglages, on a été très sensible au fait qu'on ne maitrise pas toujours le fonctionnement réel de ces modèles. On s'aperçoit ainsi que certaines fonctions d'un modèle ne réagissent pas dans les conditions pour lesquelles elles ont été créées et perdent donc leur intérêt. La procédure qui consiste à introduire de nouvelles fonctions pour remédier aux défaillances antérieures accentue encore ce phénomène et rend l'analyse du modèle très difficile. Si, au contraire, on simplifie le modèle en supprimant certaines fonctions, on constate que l'on gagne en compréhension du modèle sans perdre beaucoup sur ses performances. Dans ces conditions, dans le présent travail, on a procédé de façon inverse en partant du modèle le plus élémen- taire possible (1 réservoir, 1 paramètre). Dans les réglages et les perfectionnements de ce modèle, on s'est attaché à suivre de près son fonctionnement réel pour vérifier que les modestes complications introduites étaient justifiées.

\section{Conception du modèle}

La première idée est de concevoir un modèle à un seul réservoir qui reçoit les pluies, est soumis à l'évaporation et se vidange pour donner les débits, comme l'indique la figure 1.

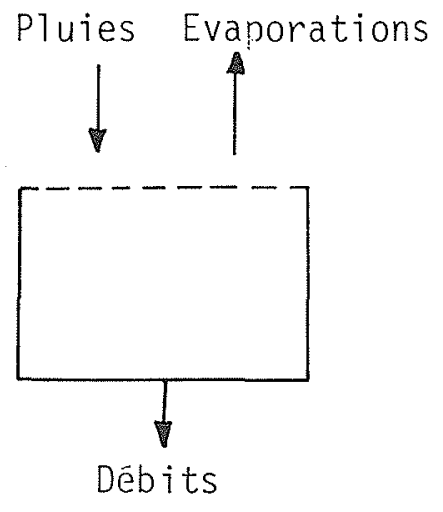

Figure 1. - Modèle à 1 réservoir

De tels modèles existent (Haan, 1972), mais l'essai sur l'Orgeval conduit à de mauvais résultats: les basses eaux sont très grossies par ce modèle et les pointes de crue sont inexistantes. Il semble nécessaire de compliquer le modèle en prenant deux réservoirs: un réservoir (S) assurant le bilan hydrologique et un réservoir (R) assurant le routage des débits. Le schéma du modèle est celui indiqué par la figure 2 .

Le réservoir superficiel $S$ reçoit une fraction $s$ de la pluie et est soumis à l'évaporation, ces deux fonctions dépendent de son niveau. La fraction complémentaire de 

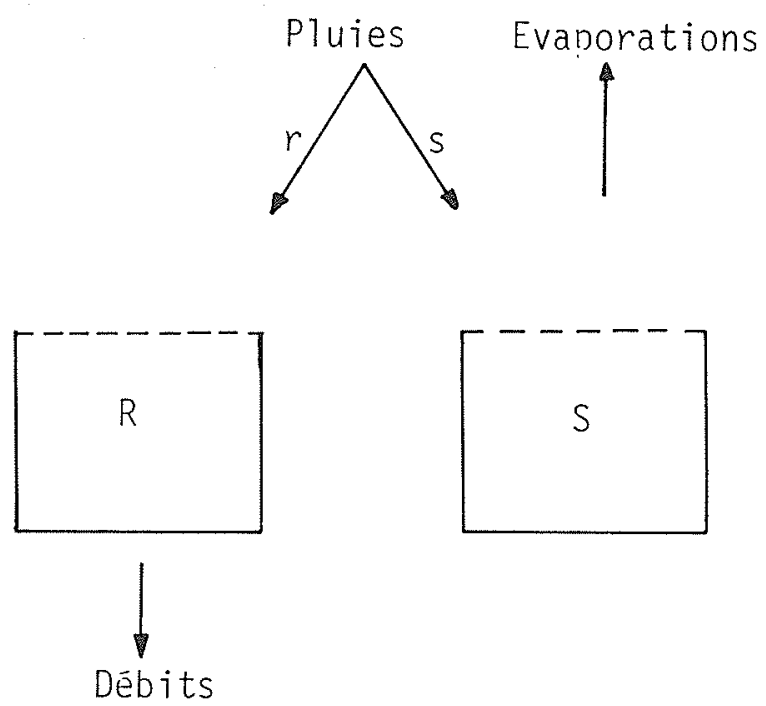

Figure 2. - Modèle étudié

la pluie $(r)$ va dans le réservoir de routage $R$. Nous allons préciser maintenant les relations analytiques choisies pour décrire les différentes fonctions de ce modèle simplifié.

\section{Fonctionnement du réservoir superficiel}

On considère que la pluie $P$ sert à faire face en priorité à $E_{p}(*)$. A la suite de cette opération, la pluie restante est notée $P^{\prime}$ et l'évaporation potentielle restante $E_{p}^{\prime}$.

Si $E_{p}>P$ on a $E_{p}^{\prime}=E p-P$ et $P^{\prime}=0$

Si $E_{p} \leqslant P$ on a $E_{p}^{\prime}=0$ et $P^{\prime}=P-E_{p}$

Le réservoir $S$ soumis à $E_{p}^{\prime}$ va donner l'évaporation réelle $E_{p}$. Il est naturel de considérer :

- que si $S=0$,

$$
E_{r}=0
$$

- et que si également $S=\alpha$ (capacité maximale),

$$
E_{r}=E_{p}^{\prime} \text {. }
$$

La relation la plus simple qui satisfait à ces deux conditions est :

$$
E_{r}=E_{p}^{\prime} \cdot S / a
$$

En ce qui concerne l'évaporation, il nous a semblé inutile d'avoir recours à des formules faisant appel à de nombreuses variables. Compte tenu du caractère très sommaire du modèle, on s'est contenté de prendre une évaporation liée uniquement à la température. Une comparaison avec l'évaporation en bac COLORADO nous a conduit à prendre au niveau journalier :

$$
\begin{array}{llll}
E_{p}=0 & \text { si } & t \leqslant 0 \\
E_{p}=\left(\frac{t}{6}\right)^{1.4} & \text { si } & t>0
\end{array}
$$

$t$ étant la température moyenne journalière exprimée en degrés Celsius.

(*) Evaporation potentielle.
On peut maintenant préciser le rôle du réservoir superficiel. Ce réservoir fonctionne comme un indice des pluies et des évaporations antérieures qui sert à fixer la proportion de pluie qui est admise à s'écouler.

Pour expliciter $r$ (et donc $s=1-r$ ), on s'est inspiré de la relation proposée depuis longtemps par le Soil Conservation Service (USDA). Ce dernier pose que le ruissellement consécutif à la pluie $P^{\prime}$ est donné par la relation

$$
\left(P^{\prime}-u\right)^{2} /\left(P^{\prime}+v\right)
$$

$u$ et $v$ étant deux paramètres tels que $v \gg u$. $P$ pour simplifier, on a pris $u=0$.

Désignons par a le niveau maximal admissible du réservoir $S$. Il parait rationnel de poser $v=a-S$ ( $S$ désignant à la fois le réservoir et son niveau). Dans ces conditions, il vient :

$$
r=P^{\prime} /\left(P^{\prime}+a-S\right)
$$

et par conséquent

$$
s=(a-S) /\left(P^{\prime}+a-S\right) .
$$

\section{Fonctionnement du réservoir de routage}

Le réservoir de routage $R$ sert au transit de la fraction $r$ de la pluie $P^{\prime}$ prédéterminée à l'écoulement. Le débit $Q$ fourni par ce réservoir $R$ est généralement exprimé comme une fonction puissance du niveau de ce réservoir. On peut hésiter à faire se produire le débit $Q$ le même jour que la pluie $P^{\prime}$, ou le jour suivant. Cela dépend du temps de réponse du bassin et la meilleure solution consisterait à utiliser un décalage précis qui ne s'exprimerait pas en un nombre entier de jours. Cela poserait des problèmes pour comparer la chronique des débits journaliers observés et celle des débits calculés. On a préféré, dans le cas présent, introduire un décalage de 1 jour. La lettre $j$ désignant la date à laquelle se réfère la variable étudiée, on aura donc:

$$
Q_{j+1}=\alpha R j^{\beta}
$$

Il est intéressant de voir comment se vidange le réservoir $R$ en l'absence de pluies. L'équation de continuité s'écrit :

soit :

$$
\begin{aligned}
& R_{j+1}=R j-Q_{j+1} \\
& R_{j+1}=R j-\alpha R j^{\beta}
\end{aligned}
$$

Selon la valeur de $\beta$ on obtient les 3 évolutions de la figure 3.

Dans le cas où $\beta<1$, la vidange est obtenue en un nombre fini d'itérations. Cela ne correspond pas à la réalité.

Avec $\beta=1$, on obtient une décroissance géométrique des débits relâchés. Cette décroissance ne correspond pas non plus aux décrues observées généralement et en particulier sur le bassin de l'Orgeval.

La solution la plus acceptable correspond à $\beta>1$, mais il faut que $R$ soit toujours inférieur à $R x$ sans quoi on obtiendrait une vidange trop brutale.

D'une manière générale, si l'on adopte une loi

$$
Q_{j+1}=f(R j)
$$




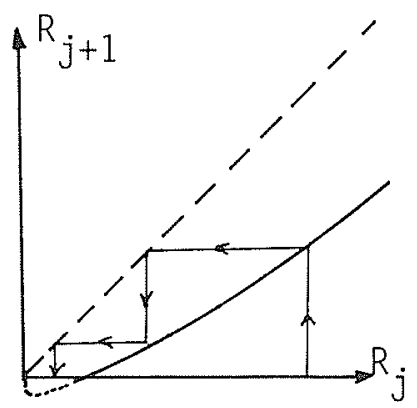

$\beta<1$

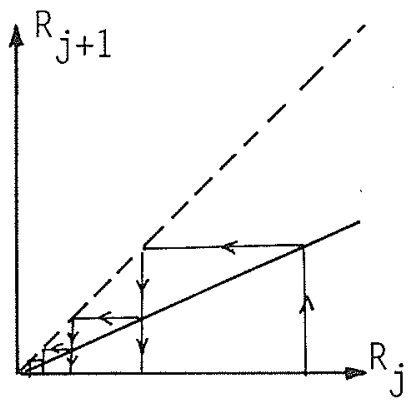

$\beta=1$

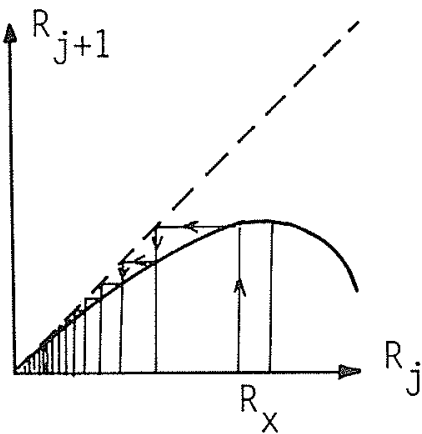

$\beta>1$

Figure 3. - Vidanges du réservoir de routage

il faut que la dérivée $f^{\prime}$ soit partout comprise entre 0 et 1 pour qu'à toute entrée corresponde une sortie qui lui soit inférieure.

Compte tenu de ce qui précède, on a retenu la loi simple suivante :

$$
Q_{j+1}=\frac{R j^{2}}{R j+b}
$$

qui conduit, en l'absence de pluies, à la vidange représentée par la figure 4.

Le paramètre $b$ représente la quantité d'eau maximale que le réservoir $R$ peut emmagasiner pour en différer l'écoulement.

\section{Contrôle du modèle}

Dans une première étape, par mesure de simplification et par référence au modele à réservoir unique dont on est parti, on prend $b=a$, c'est-à-dire un modèle à un seul paramètre. Ce modèle, représenté sur la figure 5, a été calé (paramètre $a$ ) et contrôlé.

Le calage se fait sur une période de 3 ans (19631965), la première année ne servant qu'à la mise en train du modèle (initialisation du réservoir $S$ ). Le modèle est contrôlé sur une période de 5 ans (1976-1981) débutant 11 ans après la période de calage. Pour effectuer le calage, ainsi que le contrôle, il faut pouvoir disposer d'un critère numérique qui permette de comparer des chroniques concomitantes. On a utilisé pour cela le critère très simple de l'erreur quadratique moyenne :

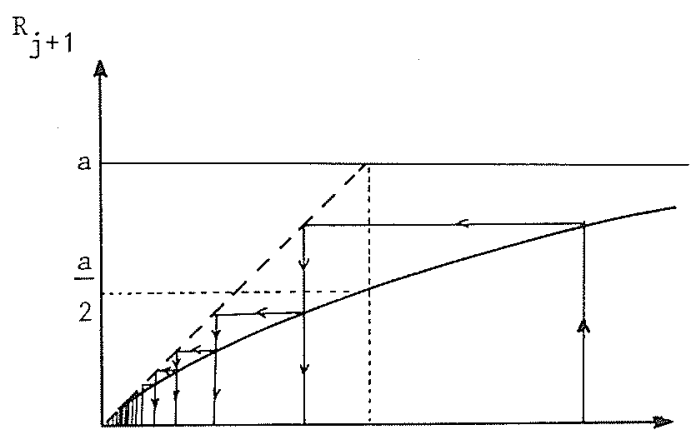

$R j$

Figure 4. - Vidange du réservoir de routage

$$
C=\left(1 / N \sum_{j=0}^{N}(Q j-Q j)^{2}\right)^{0.5}
$$

sur la période de calage, on a obtenu $a=141 \mathrm{~mm}$ et sur la période de contrôle $C=863 \mathrm{1} / \mathrm{s}(123 \% \mathrm{du}$ module).

\section{Amélioration du modèle}

Une première tentative d'amélioration du modèle a été effectuée en affectant à $R$ et à $S$ des paramètres différents : $b \neq a$.

On a obtenu:

$$
A=188 \mathrm{~mm}, \quad b=55 \mathrm{~m} \text { et } C=8761 / \mathrm{s} .
$$

Les résultats sont meilleurs sur la période de calage, mais moins bons sur la période de contrôle. Bien que

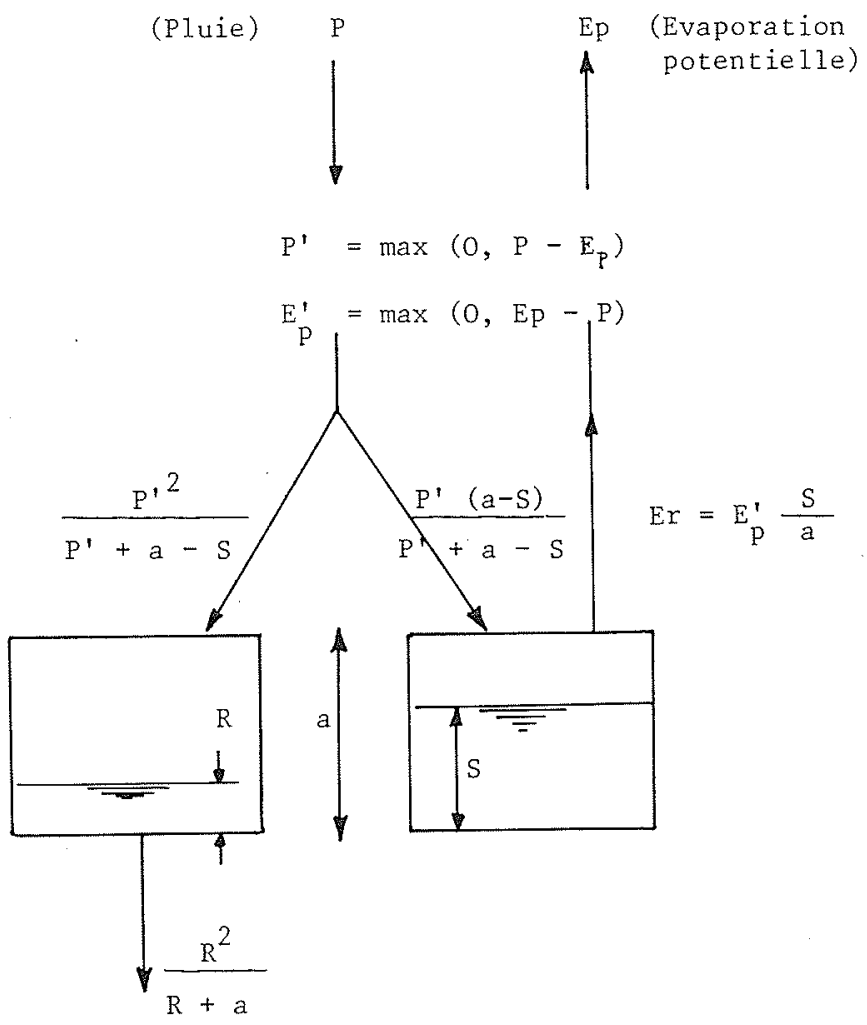

Figure 5. - Module à un paramètre. 

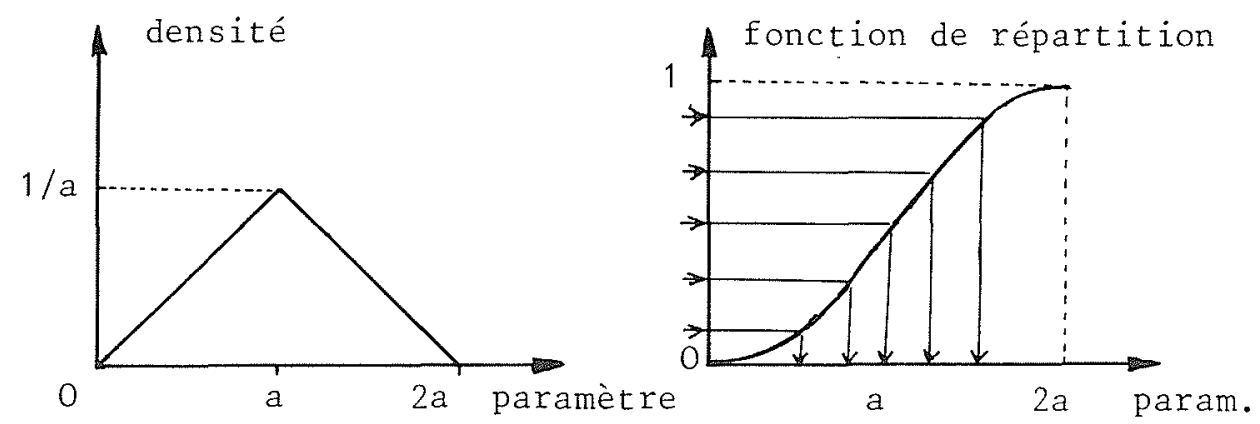

Figure 6. - Densité de répartition du paramètre du modèle

les crues soient mieux rendues, le deuxième paramètre n'est pas justifié. Une deuxième tentative a consisté à introduire de façon simple l'idée développée par J.J. Moore et R.T. Clarke (1981). Il s'agit de prendre en compte l'hétérogénéité inévitable du bassin versant en imaginant une répartition statistique de modules $(R, S)$. La répartition la plus simple consistait en une répartition à densité ayant la forme d'un triangle (fig. 6):

Dans la pratique, on utilise un nombre entier de modules, pas trop grand pour limiter le temps de calcul. L'emploi de 10 modules ne conduisant pas à plus de précision que l'emploi de 5 modules, on a utilisé un tel ensemble de 5 modules, chacun représentant un cinquième du bassin versant. Les 5 modules, fonctionnant en parallèle, ont leur paramètre obtenu par inversion de la loi précédente.

Si $i$ est le rang du module, on prend

$$
F i=\frac{i-0.3}{5+0.4}
$$

$a$ étant le paramètre global du modèle et $a_{i}$ le paramètre du ième module, on aura:

- pour $F i \leqslant 0.5 ; a_{i}=a(2 F i)^{0.5}$

- pour $F i>0.5 ; a_{i}=a\left(2-(2(1-F i))^{0.5}\right)$
On obtient ainsi : $a_{1}=0.51 a ; a_{2}=0.79 a ; a_{3}=a$; $a_{4}=1.21 a ; a_{5}=1.49 a$.

Un schéma d'ensemble apparaît sur la figure 7, dans laquelle $r_{i}$ représente:

$$
\left(a_{i}-S_{i}\right) /\left(P^{\dagger}+a_{i}-S_{i}\right)
$$

Ce modèle donne des résultats sensiblement meilleurs au calage et sur la période de contrôle : $a=154 \mathrm{~m}$ $C=8461 / \mathrm{s}$.

Compte tenu de la simplicité du modèle (un seul paramètre) et du fait que les crues sont mieux rendues que dans le modèle sans distribution, on considère cette deuxiéme tentative comme acceptable. La figure 8 montre la reconstitution de l'année 1979 au cours de la période de contrôle.

La figure 9 rassemble les résultats obtenus par les 4 modèles cités dans la note.

\section{Sensibilité du modèle}

Le critère $C$ réagit de façon univoque de part et d'autre de la valeur optimale du paramètre, aux variations de ce paramètre. Ces conditions facilitent le calage du modèle. Par contre, si l'on procède au calage du modèle à différentes périodes, on constate que le paramètre varie assez nettement d'une période à l'autre.

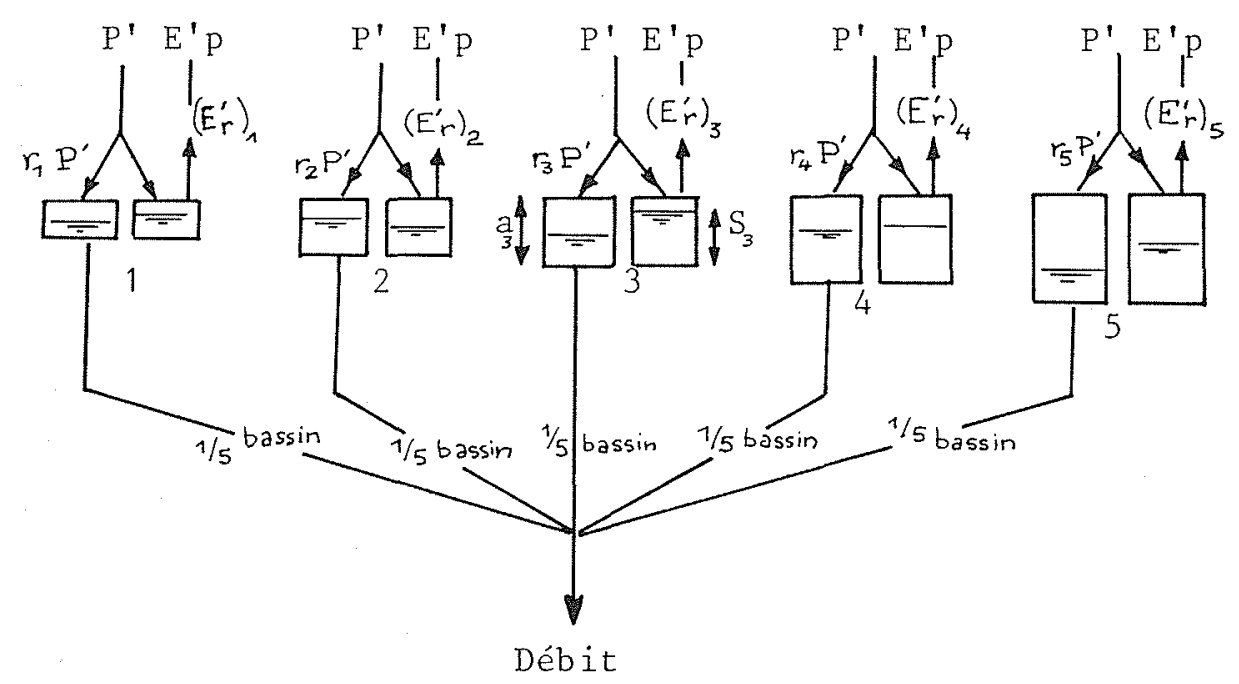

Figure 7. - Distribution des modules 


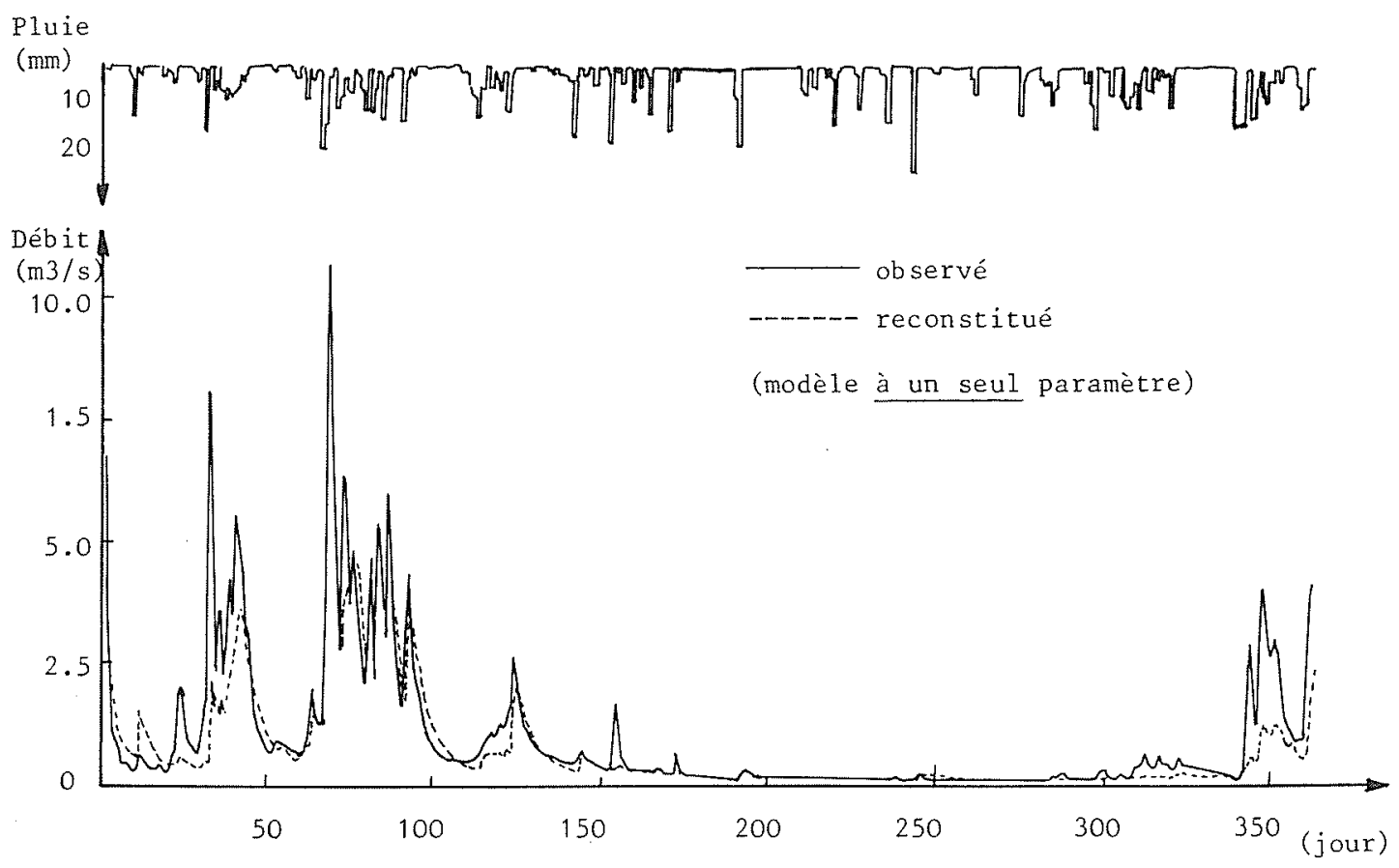

FIgure 8. - Reconstitution de l'année 1979

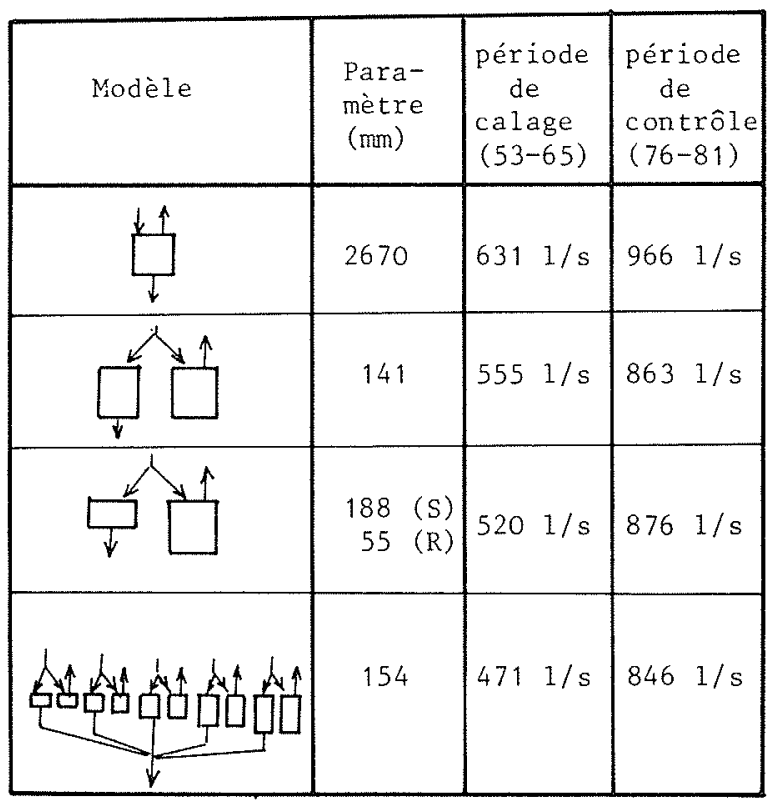

Figure 9. - Comparaison numérique des modèles. (erreur quadratique moyenne).

\section{Conclusion}

On s'est efforcé de tester les performances d'un modèle aussi simple que possible sachant, comme l'indique Naef (1981), que les complications conceptuelles ne sont pas très convaincantes en général. Il est, bien entendu, toujours préférable d'approfondir la compréhension des divergences entre un modèle et la réalité afin d'imaginer d'autres schémas plus performants, plutôt que de compliquer un modèle inadéquat par une multiplication des paramètres. 


\section{Références}

GALEA G. (1972). - Modèles déterministes des relations Pluies - Débits sur le bassin versant de l'Orgeval. Thèse de Doctorat de spécialité en Géologie Appliquée (Montpellier, 22 juin 1972).

HAAN C.T. (1972). - A water yield model for small watersheds. Water Resources Research, Vol 8, $\mathrm{n}^{\circ}$ 1, Feb. 72, pp. 58-69.

MOORE R.J., and CLARKE R.T. (1981). - A distribution Function Approach to Rainfall Runoff Modeling. Water Resources Research, Vol 17, n 5, Oct. 81, pp. 1367-1382.

NAEF F. (1981). - Can we model the rainfall - runoff process today ? Hydrological Sciences Bulletin, 26, 3, 9/1981, pp. 281-289.

OBERLIN G. (1978). - L'évapotranspiration réelle en hydrologie. S.H.F., XV Journées de l'Hydraulique, Toulouse, 5-7 septembre 1978, Question I, Rapport 4.

RIFAAT S. (1980). - Comparaison de plusieurs techniques de reconstitution de débits moyens mensuels. Mémoire de $3^{\mathrm{e}}$ Cycle d'Hydrologie, Ecole Polytechnique Fédérale de Lausanne, Octobre 1980.

TIERCELIN J R, et LEVIANDIER T. (1977). - Un modèle pour la simulation des débits journaliers: le modèle Dejф̆reg. Mémoire $\mathrm{n}^{\circ}$ 8, Février 1977, Cemagref, Ministère de l'Agriculture/ $\mathrm{La}$ Technique de l'Eau et de l'Assainissement, n' 377, mai 1978. 